\title{
Experimental modulation of external microbiome affects nestmate recognition in harvester ants (Pogonomyrmex barbatus)
}

Andy Dosmann, Nassim Bahet, Deborah M Gordon

Social insects use odors as cues for a variety of behavioral responses, including nestmate recognition. Past research on nestmate recognition indicates cuticular hydrocarbons are important nestmate discriminators for social insects, but other factors are likely to contribute to colony-specific odors. Here we experimentally tested whether external microbes contribute to nestmate recognition in red harvester ants (Pogonomyrmex barbatus). We changed the external microbiome of ants through topical application of either antibiotics or microbial cultures. We then observed behavior of nestmates when treated ants were returned to the nest. Ants whose external microbiome was augmented with microbial cultures were much more likely to be rejected than controls, but ants treated with antibiotics were not. This result is consistent with the possibility that external microbes are used for nestmate recognition. 
1 Title Page: Experimental modulation of external microbiome affects nestmate recognition in

2 harvester ants (Pogonomyrmex barbatus)

3 Dr. Andy Dosmann*

4 Department of Natural Sciences

5 Minerva Schools at Keck Graduate Institute

6 San Francisco, CA

$7 \quad$ Nassim Bahet

8 Stanford University Online High School

9 Stanford, CA

10 Dr. Deborah M. Gordon

11 Department of Biology

12 Stanford University

13 Stanford, CA

$14 *$ - Corresponding author information:

1524 Seymour St., Middlebury, VT 05753, USA

16 dosmann1@gmail.com

17 Experimental modulation of external microbiome affects nestmate recognition in harvester ants

18 (Pogonomyrmex barbatus) 
19 Dosmann, A, Bahet, N., and Gordon, D. M.

\section{ABSTRACT}

21 Social insects use odors as cues for a variety of behavioral responses, including nestmate

22 recognition. Past research on nestmate recognition indicates cuticular hydrocarbons are important

23 nestmate discriminators for social insects, but other factors are likely to contribute to colony-

24 specific odors. Here we experimentally tested whether external microbes contribute to nestmate

25 recognition in red harvester ants (Pogonomyrmex barbatus). We changed the external

26 microbiome of ants through topical application of either antibiotics or microbial cultures. We then

27 observed behavior of nestmates when treated ants were returned to the nest. Ants whose external

28 microbiome was augmented with microbial cultures were much more likely to be rejected than

29 controls, but ants treated with antibiotics were not. This result is consistent with the possibility

30 that external microbes are used for nestmate recognition.

31 Keywords: Nestmate recognition, microbiome, harvester ants

32 INTRODUCTION

33 In ants, individuals discriminate between nestmates and non-nestmates, which is often equivalent

34 to kin discrimination, by using chemical cues (Vander Meer and Morel 1998; van Zweden and 35 d'Ettorre 2010; Sturgis and Gordon 2012). Cuticular hydrocarbons (CHCs) are chemical 
36 compounds that serve a variety of functions in social insects but very often are implicated in

37 nestmate recognition by both empirical and theoretical research (Wagner et al. 2000, Howard and

38 Blomquist 2005). CHCs are synthesized from lipids, and the CHC profile of an ant, which

39 consists of various classes of alkanes, alkenes and methyl-branched hydrocarbons in differing

40 proportions, can be both task and colony specific (Sturgis and Gordon 2012). In Cataglyphis

41 niger, when hydrocarbons are isolated from the postpharyngeal gland and applied to one of a pair

42 of live ants, the untreated ant behaves in a way that indicates CHCs function as nestmate

43 discriminators (Lahav et al. 1999). Esponda and Gordon (2015) recently proposed a model of

44 distributed nestmate recognition in which each ant in a colony has a flexible decision boundary in

45 multidimensional odor space, acquired through interactions, that divides nestmates from non-

46 nestmates.

47 For good reason, research on nestmate recognition in social insects has focused on CHCs, but

48 other chemicals are also likely to play a role in nestmate recognition (Sturgis and Gordon 2012).

49 Davis et al. (2013) reviewed studies showing that volatile chemicals emitted by microbes affect

50 insect behavior ranging from foraging to oviposition. Lizé et al. (2014) showed that bacteria

51 influence kin recognition in Drosophila melanogaster. Although CHCs alone are sufficient for

52 nestmate recognition in some species of ants (Lahav et al. 1999; Wagner et al. 2000), this does

53 not preclude a role for microbes. Both internal and external microbial communities are colony-

54 specific in ants (Anderson et al. 2013; Hu et al. 2014), and thus potential nestmate discriminators.

55 Over ten years ago, Matsuura (2001) experimentally showed that bacteria mediate nestmate

56 recognition in termites (Reticulitermes speratus). However, the role of microbes in nestmate

57 recognition of ants has not been investigated, except for a study by Richard et al. (2007) showing

58 that ants from one colony of fungus growing ants (Acromyrmex spp.), if reared on a second

59 colony's fungus, experienced less aggression when introduced to the second colony. 
60 Here we test the hypothesis that the microbial composition on an ant's cuticle affects whether it is

61 treated as a nestmate. Both pathogenic and protective microbes inhabit the surface of insect

62 cuticles (Douglas 2015), and they could produce chemicals that affect nestmate recognition

63 (Davis et al. 2013). Previous work in honey bees (Apis mellifera) showed that internal injection

64 of bacteria caused significant changes in $\mathrm{CHC}$ profiles, and in turn nestmate recognition (Richard

65 et al. 2012), but here we isolate the effect of external microbes on nestmate recognition. To test

66 the hypothesis that the external microbiome affects nestmate recognition in ants, we

67 experimentally modulated the microbes on the cuticles of red harvester ants (Pogonomyrmex

68 barbatus) and tested whether those ants were rejected by their nestmates. P. barbatus can

69 distinguish between nestmates and non-nestmates using CHCs alone (Wagner et al. 2000), but

70 microbes may still play a role. We predicted that if the external microbiome plays a role in

71 nestmate recognition, individuals with an altered external microbiome would not be accepted as

72 nestmates when returned to their nest.

73 METHODS

74 Study species

75 Experiments were conducted in 3 queenright, brood-producing laboratory colonies of red

76 harvester ants (Pogonomyrmex barbatus) obtained from a field site near Rodeo, NM. Details of

77 lab conditions are found in Gordon et al. (2005).

78 Assay of nestmate recognition 
79 On the day of the experiment, we first removed a group of ants from their nest's foraging arena

$80 \sim 4$ hours prior to behavioral testing. Group size (9-20 ants) depended on the number of ants

81 active in the foraging arena during sampling. Each ant then received one of five experimental or

82 control treatments, described below, and was painted with a color corresponding to its treatment

83 for identification during behavioral observation (Gordon et al. 2005). We then returned the group

84 of treated and marked ants to a location near the nest entrance, where they interacted with

85 resident nestmates. We performed the test with groups of ants at the nest entrance, rather than

86 paired individuals in a foreign arena, because recent models indicate that accurate nestmate

87 recognition occurs at the colony level (Johnson et al. 2011; Esponda and Gordon 2015) and

88 empirical work indicates that testing ants in groups provides results that are more consistent and

89 indicative of natural responses than tests with individuals (Roulston et al. 2003). An observer

90 blind to the treatment groups then scored whether each ant was rejected from the nest during the

91 following 20 minutes. Rejection, which was often preceded by aggressive acts like biting,

92 consisted of a resident ant seizing a treated ant and carrying it out of the nest or away from the

93 nest entrance towards the midden pile at the far end of the foraging arena.

94 Experimental treatment

95 We manipulated the external microbiome of ants $(\mathrm{N}=111)$ using five different treatments: 1$)$

96 Antibiotic group: To decrease the external microbiome, we coated each ant's body surface with a

97 1\% rifampin/sterile water solution. 2) Microbial group: To increase the external microbiome, we

98 coated each ant's body surface with a solution of Lysogeny Broth (LB) broth (Becton Dickenson,

99 Franklin Lakes, NJ) that had previously been cultured from nestmates. Because only a small

100 proportion of the microbiome survives in culture (Rappe and Giovannoni 2003), this solution

101 modified and augmented an ant's external microbiome, and we then confirmed this change (see 
102 below). In the control groups, we treated ants with 3) Water: sterile water, 4) Broth: sterile LB

103 broth, or 5) Nest: simply removed ants from the nest and marked them before return.

\section{Validation of treatment}

105 To validate that our treatment produced the intended effect on the ants' external microbiome, we 106 used the method of Ren et al. (2007) to culture the external microbiome of additional ants $(\mathrm{N}=$

107 35). Although only a small proportion of microbial species can be cultured (Rappe and

108 Giovannoni 2003), we used differences in the amount of culturable microbes obtained, measured 109 by counting the number of microbial colony forming units (CFUs) that grew on LB agar plates, to

110 indicate effectiveness of the treatments. A greater number of CFUs indicates a greater amount of

111 external microbes on an ant.

112 Effect of treatment on survival

113 To determine whether the treatments had any beneficial or adverse effects, we treated additional

114 ants $(\mathrm{N}=72)$ and kept them in Petri dishes with water for two weeks. We checked on individuals 115 once daily and recorded whether the ant was alive or dead.

116 Statistical analysis

117 To control for non-independence produced by colony membership and returning ants in groups

118 (i.e. block design), we used a generalized linear mixed-effects model fitting block and colony as

119 random effects, with treatment (Microbial, Antibiotic, LB Broth, Water, Nest) as a categorical 120 predictor and rejection as a binary dependent variable. We used the lmer function in the $l m e 4$ 
121 package in R 3.0.2 for linear mixed-effects models, and the multcomp package to make Tukey

122 post hoc comparisons of treatment effects.

123 Because the counts of CFUs were nonparametrically distributed, we used Wilcoxon Rank Sum

124 Tests to assess the treatment validation. We made planned comparisons that would indicate

125 treatment efficacy (Antibiotic vs. Broth, Water, and Nest; Microbial vs. Broth, Water, and Nest),

126 correcting alpha for the false discovery rate according to Benjamini and Hochberg (1995).

127 To determine whether an experimental treatment significantly increased or decreased survival, we

128 used a Cox proportional hazards model with the survival package in R (Crawley 2007), and used

129 the multcomp package to make Tukey post hoc comparisons of treatment effects.

130 RESULTS

131 The experimental treatment modulated ants' external microbiome, as measured by number of

132 colony forming units. The topical application of the microbial culture significantly increased in

133 the number of CFUs obtained from an ant's cuticle compared to each of the controls (Microbial

134 vs. Broth: $W=49, P=0.002, N=10$, vs. Water: $W=40.5, P=0.006, N=9$, vs. Nest: $W=49, P$

$135=0.002, N=10)$. Antibiotic treatment significantly decreased the number of CFUs compared to

136 controls, except for the water treatment (Antibiotic vs. Broth: $W=7.5, P=0.035, N=10$; vs.

137 Water: $W=13.5, P=0.317, N=9$; vs. Nest: $W=7, P=0.026, N=10$ ). With 5 of 6 comparisons

$138 P<0.05$, our alpha, controlled for false discovery rate, is 0.042 .

139 Changes in an ant's external microbiome influenced whether it was rejected from its nest by

140 nestmates (Figure 1). Ants with experimentally augmented external microbiomes were more 
141 likely to be rejected than ants treated with broth alone, sterile water, or only marked (all post hoc

142 comparisons $|\mathrm{Z}|>3.8, P<0.001)$. Ants treated with the antibiotic solution were not more likely

143 to be rejected than ants treated with broth alone, sterile water, or only marked (all post hoc

144 comparisons $|\mathrm{Z}|<1.1, P>0.80)$.

145 Ants in the Microbial group died significantly sooner than ants given the water treatment $(\mathrm{Z}=$

$146-3.085, P=0.017)$. There were no significant differences in survival between the Microbial group

147 and the other control groups (all post hoc comparisons $|\mathrm{Z}|<1.64, P>0.46$ ), or between the

148 Antibiotic group and the control groups (all post hoc comparisons $|\mathrm{Z}|<1.173, P>0.76$ ).

\section{DISCUSSION}

150 We found that as we predicted, ants treated with additional microbes were rejected by resident

151 colony members as if they were non-nestmates. This aligns with our predictions and supports our 152 hypothesis that the external microbiome plays a role in nestmate recognition in P. barbatus.

153 Contrary to predictions, ants treated with the antibiotic solution were not rejected by resident

154 colony members. Because the antibiotic treatment was delivered in a sterile water solution, ants'

155 CHC profiles were likely unaltered, in which case many familiar odors would persist. This is

156 consistent with the possibility that the presence of a foreign odor is more likely to lead to the

157 recognition of a non-nestmate than absence of a familiar odor (van Zweden and d'Ettorre 2010;

158 Esponda and Gordon 2015), as well as experimental data showing that carpenter ants

159 (Camponotus herculeanus) reject non-nestmates by recognizing novel CHCs (Guerrieri et al.

160 2009). 
161 The rejection of ants with augmented external microbiomes could be interpreted as a form of

162 social immunity (Cremer et al. 2007), in which ants with an unfamiliar or unusual microbiome

163 are perceived as sick nestmates in need of quarantine. Social immunity may involve a

164 microbially-mediated form of nestmate recognition, since a microbe that causes an individual to

165 be placed on the 'reject' side of another ant's boundary may or may not be a pathogen. In this

166 way, social immunity could constitute an additional function of microbially-mediated nestmate

167 recognition. We found a significant difference in survival between ants from the Microbial group

168 and those treated with water, but not between ants from the Microbial group and those from the

169 Broth and Nest groups. Thus, the extent to which the microbial treatment may have included

170 pathogens is not resolved and we do not know the extent to which rejection of treated ants

171 contributed to social immunity.

172 In addition to further research on social immunity, our results suggest several research directions

173 that may provide insight on the role of microbes in chemical communication. First, it will be

174 important to learn what microbial species are key to nestmate recognition and what chemicals

175 they produce. In hyenas, fermentative bacteria appear to play a key role in chemical

176 communication (Theis et al. 2013). Second, it will be important to establish how much microbial

177 communities differ among colonies, and how consistent those communities are over time. In

178 leafcutter ants (Acromyrmex echinatior), the external microbiome is colony-specific and

179 differences are maintained up to 10 years, even after colonies were kept in laboratory conditions

180 (Anderson et al. 2013). Third, an ant's microbiome may be task specific, as well as colony-

181 specific. In P. barbatus, CHCs are task specific (Sturgis and Gordon 2013) and influence task

182 allocation (Greene and Gordon 2003). It is likely that microbial communities of ants vary with

183 task type, as foragers are exposed to different microbes from brood care or midden workers 
184 (Cremer et al. 2007). Microbes may also inform task decisions alongside CHCs, or could directly

185 affect the structure of CHCs.

186 In conclusion, our results support a role for microbes in nestmate recognition in harvester ants.

187 They align with experimental data showing microbes influence nestmate/non-nestmate

188 recognition in termites (Matsuura 2001) and leaf-cutting ants (Richard et al. 2007), and show that

189 external microbes alone can impact nestmate recognition. This finding augments extensive

190 evidence of CHC-mediated nestmate recognition in social insects (Vander Meer and Morel 1998;

191 van Zweden and d'Ettorre 2010; Sturgis and Gordon 2012), and calls for further investigation of

192 the role of microbes in chemical communication of social insects.

193 REFERENCES

194 Anderson SB, Hansen LH, Sapountzis P, Sorensen SJ, Boomsma JJ (2013) Specificity and

195 stability of the Acromyrmex-Pseudonocardia symbiosis. Mol Ecol 22:4307-4321

196 Benjamini Y, Hochberg Y (1995) Controlling the false discovery rate: a practical and powerful

197 approach to multiple testing. J R Stat Soc 57:289-300

198 Crawley MJ (2007) The R Book. John Wiley \& Sons.

199 Cremer S, Armitage SAO, Schmid-Hempel (2007) Social Immunity. Curr Biol 17:R693-R702

200 Davis TS, Crippen TL, Hofstetter RW, Tomberlin JK (2013) Microbial Volatile Emissions as

$201 \quad$ Insect Semiochemicals. J Chem Ecol 39:840-859

202 Douglas AE (2015) Multiorganismal Insects: Diversity and Function of Resident

203 Microorganisms. Ann Rev Entomol 60:17-34 
204 Esponda F, Gordon DM (2015) Distributed nestmate recognition in ants. Proc R Soc B 282:1806

205 Gordon DM, Chu J, Lillie A, Tissot M, Pinter N (2005) Variation in the transition from inside to 206 outside work in the red harvester ant Pogonomyrmex barbatus. Insect Soc 52:212-217.

207 Greene MJ, Gordon DM (2003) Social insects: Cuticular hydrocarbons inform task decisions. $208 \quad$ Nature 423:32.

209 Guerrieri FJ, Nehring V, Jorgensen CG, Nielsen J, Galizia CG, d'Ettorre P (2009) Ants recognize 210 foes not friends. Proc R Soc B 276:2461-2468.

211 Howard RW, Blomquist GJ (2005) Ecological, behavioral, and biochemical aspects of insect 212 hydrocarbons. Ann Rev Entomol 50:371-393

$213 \mathrm{Hu}$ Y, Lukasik P, Moreau CS, Russell JA (2014) Correlates of gut community composition across 214 an ant species (Cephalotes varians) elucidate causes and consequences of symbiotic 215 variability. Mol Ecol 23:1284-1300

216 Johnson BR, van Wilgenburg E, Tsutsui ND (2011). Nestmate recognition in social insects: 217 overcoming physiological constraints with collective decision making. Behav Ecol $218 \quad$ Sociobiol 65:935-944.

219 Lahav S, Soroker V, Hefetz A, Vander Meer RK (1999) Direct Behavioral Evidence for 220 Hydrocarbons as Ant Recognition Discriminators. Naturwissenschaften 86:246-249

221 Lizé A, McKay R, Lewis Z (2014) Kin recognition in Drosophila: the importance of ecology and 222 gut microbiota. ISME J 8:469-477

223 Matsuura K (2001) Nestmate Recognition Mediated by Intestinal Bacteria in a Termite, $224 \quad$ Reticulutermes speratus. Oikos 92:20-26

225 Rappe MS, Giovannoni SJ (2003) The uncultured microbial majority. Ann Rev Microbiol 226 $57: 369-394$

227 Ren C, Webster P, Finkel SE, Tower J (2007) Increased Internal and External Bacterial Load 228 during Drosophila Aging without Life-Span Trade-Off. Cell Metab 6:144-152. 
229 Richard FJ, Poulson M, Hefetz A, Errard C, Nash DR, Boomsma JJ (2007) The origin of the 230 chemical profiles of fungal symbionts and their significance for nestmate recognition in 231 Acromyrmex leaf-cutting ants. Behav Ecol Sociobiol 61:1637-1649.

232 Roulston TH, Buczkowski G, Silverman J (2003) Nestmate discrimination in ants: Effect of 233 bioassay on aggressive behavior. Insectes Sociaux 9:73-85.

234 Sturgis SJ, Gordon DM (2012) Nestmate recognition in ants (Hymenoptera: Formicidae): a 235 review. Myrmecological News 16:101-110

236 Sturgis SJ, Gordon DM (2013) Aggression is task dependent in the red harvester ant

237 (Pogonomyrmex barbatus). Behav Ecol 24:532-539

238 van Zweden JS, d'Ettorre P (2010) Nestmate recognition in social insects and the role of 239 hydrocarbons. Insect hydrocarbons: biology, biochemistry and chemical ecology 11:222$240 \quad 243$

241 Theis KR, Vankataraman A, Dycus JA, Koonter KD, Schmitt-Matzen EN, Wagner AP, Holekamp 242 KE, Schmidt TM (2013) Symbiotic bacteria appear to mediate hyena social odors. PNAS $243 \quad 110: 19832-19837$

244 Vander Meer RK, Morel L (1998) Nestmate Recognition in Ants. In Pheromone Communication 245 in Social Insects. Westview Press, Boulder, CO.

246 Wagner D, Tissot M, Cuevas W, Gordon DM (2000) Harvester ants utilize cuticular hydrocarbons 247 in nestmate recognition. J Chem Ecol 26:2245-2257 
248 FIGURE 


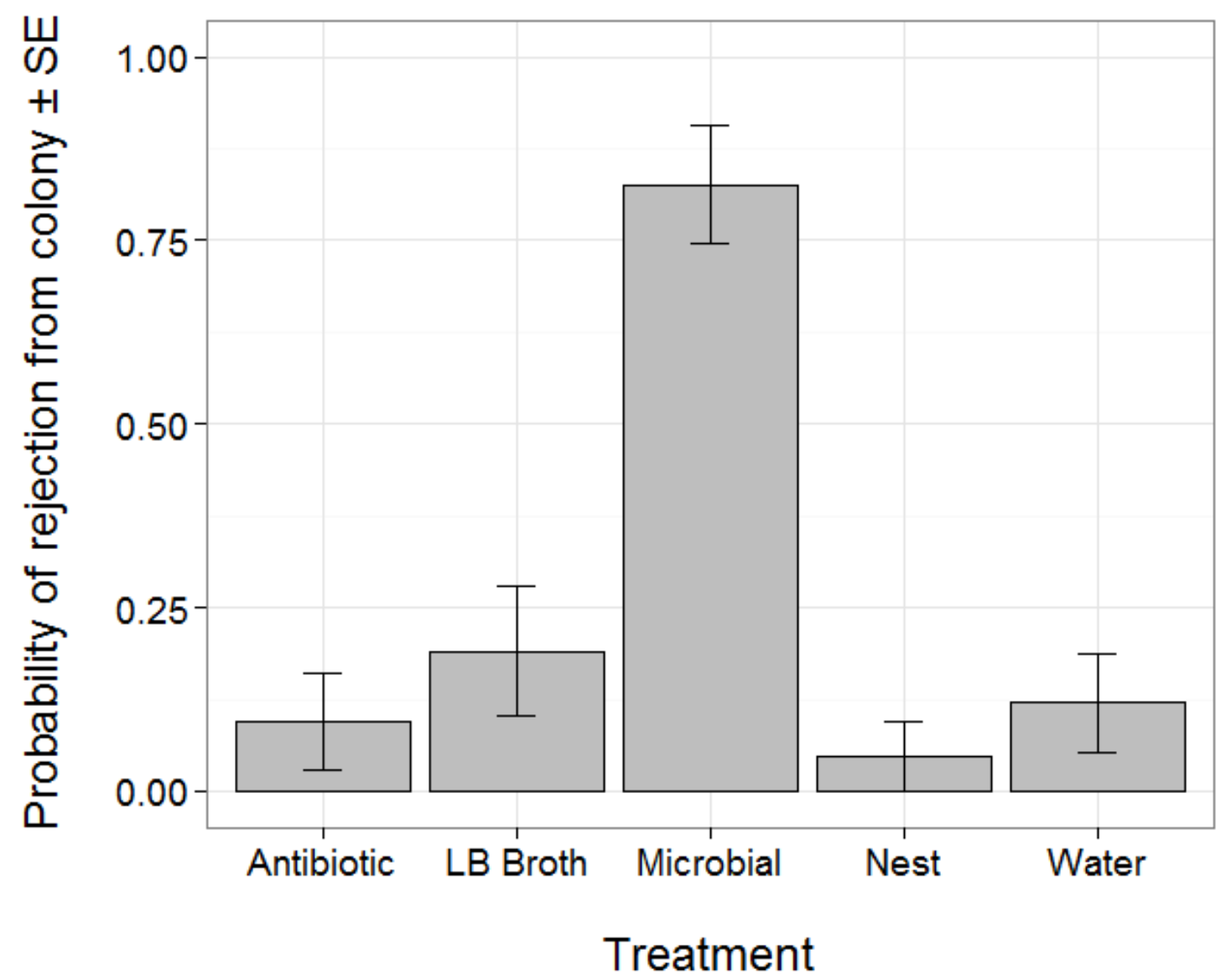

249 Figure 1 - Probability that an ant was rejected by its nestmates (i.e. not recognized as a nestmate) 250 after manipulation of its external microbiome. Antibiotic $=$ topical treatment with $1 \%$ rifampin 251 solution. LB Broth $=$ topical treatment with sterile LB broth. Microbial = topical treatment with 252 microbes cultured from ants in LB broth. Nest = no topical treatment; individuals were only 253 marked before return to nest. Water $=$ topical treatment with sterile water. 